livraisons

d'Histoire

de l'Architecture

\section{Livraisons de l'histoire de l'architecture}

$37 \mid 2019$

L'architecture mise à l'examen

\title{
« Projet de construction » aux Beaux-Arts de Téhéran, un métissage pédagogique
}

'Construction project' and the hybrid architectural education at the Fine Arts Faculty of Tehran

"Konstruktionsprojekte" an den Beaux-Arts von Teheran, ein pädagogisches Konglomerat

Shahram Abadie

\section{OpenEdition}

Journals

Édition électronique

URL : http://journals.openedition.org//ha/1392

DOI : $10.4000 /$ lha.1392

ISSN : 1960-5994

Éditeur

Association Livraisons d'histoire de l'architecture - LHA

Édition imprimée

Date de publication : 15 juin 2019

Pagination : 73-84

ISSN : 1627-4970

Référence électronique

Shahram Abadie, « «Projet de construction » aux Beaux-Arts de Téhéran, un métissage pédagogique », Livraisons de l'histoire de l'architecture [En ligne], 37 | 2019, mis en ligne le 02 janvier 2021, consulté le 25 janvier 2021. URL : http://journals.openedition.org/lha/1392 ; DOI : https:// doi.org/10.4000//ha.1392 


\section{«LE PROJET DE CONSTRUCTION » AUX BEAUX-ARTS DE TÉHÉRAN, UN MÉTISSAGE PÉDAGOGIQUE}

\section{Une faculté à la croisée des modèles européens}

Lors de la création des premiers établissements d'enseignement - académique de l'architecture en Iran, entre les deux guerres, deux « écoles » rivalisent d'influence. Un modèle plutôt d'influence allemande est d'abord proposé par Karim Taherzadeh Behzad (1888-1963), architecte formé aux Beaux-Arts d'Istanbul, puis à la Bauakademie de Berlin entre 1921 et $1926^{1}$. Suivant son projet, le ministère des Sciences établit une "école d'ingénierie et d'architecture » en 1927, au sein de l'unique académie iranienne de l'époque, Darol Fonoun (fondée en 1851), pour proposer un cursus d'ingénieur architecte en quatre ans et un autre d'assistant architecte (technicien) en deux ans. L'institution échoue au bout de deux ans, faute de financement. Une dizaine d'années plus tard, l'École supérieure d'architecture est créée par le ministère des Arts et métiers, sur le modèle français. Elle est portée par des architectes diplômés par le gouvernement (DPLG) : Mohsen Foroughi (1907-1983) et Roland Dubrulle (1907-1983), sous l'égide de leur confrère aîné André Godard (1881-1965), influent chef du service des Antiquités et d'archéologie de l'Iran depuis $1928^{2}$. Même si Taherzadeh Behzad participe, au départ, à l'équipe enseignante de la nouvelle école, le "modèle Beaux-Arts » finit bientôt par s'y imposer et provoquer son départ $^{3}$. Cet établissement fusionne, à partir de septembre 1940, avec les ateliers de peinture et de sculpture de l'École supérieure d'art pour constituer la Faculté des Beaux-Arts au sein de l'Université de Téhéran nouvellement créée (1934), et reste l'unique centre de formation des architectes en Iran pendant deux décennies.

Sous l'impulsion de son premier directeur, l'architecte français André Godard, la faculté des Beaux-Arts de Téhéran calqua son enseignement de l'architecture sur

1. Bijan Shafei, Sohrab Soroushiani et Victor Daniel, Architecture de Karim Taherzadeh Behzad [trad. du titre persan], Téhéran, Éditions Did, 2005.

2. Sur ce médiateur entre les cultures française et iranienne voir Victor Daniel, Bijan Shafei et Sohrab Soroushiani, André Godard, Téhéran, Groupe d'architectes de l'ère de l'évolution en Iran, 2015; Sarah Piram, « S'approprier un modèle français en Iran ? L’architecte André Godard (1881-1965) et la conception des musées iraniens ", Les Cahiers de l'École du Louvre, $\mathrm{n}^{\circ} 11,2017$, disponible en ligne : http://journals.openedition.org/cel/825.

3. Gholamreza Kobari, Mémoire du passé [trad. du titre persan], Téhéran, 2006, cité par Hossein Soltanzadeh, "Genèse de la faculté des Beaux-Arts" [trad. du titre persan], Memari va farhang (Architecture et culture), revue trimestrielle, vol. 10, nº 32, 2008, p. 6. 
le modèle de l'École nationale supérieure des Beaux-Arts (ENSBA) de Paris ${ }^{4}$. Un cursus composé principalement de deux étapes, premier et deuxième cycles, équivalents aux seconde et première classes parisiennes, conduit au projet de diplôme. Comme à Paris, on y reçoit une formation en atelier et par émulation ${ }^{5}$, les études sont scandées d'épreuves d'esquisse et de décoration préparées en une seule journée, des projets d'architecture sont présentés au bout d'un mois et demi de travail, en parallèle avec des cours théoriques. Tous ces enseignements sont évalués par un système de distribution de médailles et de mentions - des mots français repris tels quels dans la terminologie de la faculté - dont chacune représente entre une et trois "valeurs » ${ }^{6}$. La validation de chaque année et de chaque cycle est conditionnée par l'acquisition d'un certain nombre de valeurs, une dizaine en était par exemple requise, dans les premières années, pour accéder au cycle supérieur des études. Un moment crucial dans ce parcours est le passage du premier au second cycle, soumis à une épreuve dite "projet de construction ", encore une expression française entrée dans le jargon des étudiants iraniens des premières promotions. Le format de cet examen se rapproche d'un concours dans la mesure où les projets sont évalués par le jury de la faculté pour remporter la première ou seconde mention voire la médaille, celles-ci pouvant quelquefois être accompagnées des félicitations des professeurs. En revanche, pas de programme commun, contrairement aux concours habituels d'architecture ; chaque étudiant doit choisir son propre programme et le faire valider par le professeur de construction, avant de concevoir le projet au sein de son atelier, sans toutefois bénéficier des corrections du patron, et le présenter enfin à l'une des séances de jugement. Le projet de construction correspond ainsi à un contrôle des compétences et des connaissances de l'étudiant au bout de trois années d'études, mais également à une affirmation de la posture d'architecte par la définition autonome d'un programme.

4. Pour une présentation synthétique et comparative voir Peyman Akhgar, "L'École des Beaux-Arts de Paris et l'institutionnalisation de l'enseignement de l'architecture en Iran (1940-1968) ", HEnsA20, cahier $n^{\circ} 4$, juin 2018, p. 45-49, disponible en ligne : https://chmcc.hypotheses.org/2544.

5. Voir à ce sujet Guy Lambert, "La pédagogie de l'atelier dans l'enseignement de l'architecture en France aux XIX ${ }^{\mathrm{e}}$ et $\mathrm{XX}^{\mathrm{e}}$ siècles, une approche culturelle et matérielle ", Perspective, $\mathrm{n}^{\circ} 1,2014$, disponible en ligne : http://journals.openedition.org/perspective/4412.

6. Gholamreza Kobari, "Une mémoire du passé » et Asghar Saed Samiee (Samii), "Organisation du système pédagogique de la faculté des Beaux-Arts " [trad. des titres persans des entretiens], Memari va farhang (Architecture et culture), revue trimestrielle, vol. 10, $\mathrm{n}^{\circ} 32,2008$, respectivement p. 11-13 et 30-33; cette livraison de la revue consacrée à l'histoire orale de la faculté des Beaux-Arts de Téhéran, offre plusieurs entretiens avec les anciens élèves et enseignants que nous exploitons dans cette étude. 


\section{Projets de construction de l'atelier Seyhoun}

Une sélection des travaux d'étudiants de la faculté des Beaux-Arts, comportant une quarantaine de "projets de construction", a été publiée en $2015^{7}$. Ils proviennent des archives léguées à la bibliothèque de la faculté par Houshang Sanei (1921-2013), élève architecte de la première promotion, puis professeur de construction et directeur adjoint de l'établissement. Ces projets datent des trois premières décennies d'existence des Beaux-Arts de Téhéran, période où l'enseignement de l'architecture est dispensé exclusivement dans cette faculté, sous la direction successive d'André Godard, de Mohsen Foroughi puis de Houshang Seyhoun (1920-2014), tous trois architectes DPLG. Des protestations étudiantes et le changement du doyen de l'université conduisirent ensuite un diplômé de l'université florentine, Dariush Mirfendereski (1931-2009) à la tête de la faculté, entraînant, à compter de 1969, un revirement dans l'enseignement de l'architecture en Iran. Ces planches témoignent donc de l'ère la plus faste de l'application du "modèle Beaux-Arts " à Téhéran. À travers l'analyse d'une partie de ces travaux, la présente étude interroge l'assimilation ou l'appropriation par les étudiants et professeurs iraniens des principes de l'enseignement par le concours de l'École parisienne. Pour ce faire, quatre critères représentant les forces ou faiblesses du système Beaux-Arts sont privilégiés : la présentation des planches (langue, techniques de dessin et du rendu), le programme (en liens avec l'actualité architecturale), l'implantation du projet dans un site réel et la conception technique et constructive. L'étude est centrée sur la vingtaine de projets primés dans les concours de construction entre 1953 et 1968 par les élèves de l'atelier Seyhoun, l'un des quatre ateliers de la faculté. Le patron éponyme se prévalait d'une double formation, celle suivie au sein même des Beaux-Arts de Téhéran (1940-1945), complétée par le cursus de la première classe à Paris, dans l'atelier Zavaroni, de mai 1946 à juin 1948. Il présenta pour son diplôme, le projet du mausolée d'Avicenne (980-1037), par ailleurs lauréat du concours national lancé pour la reconstruction du tombeau du savant perse et réalisé de 1949 à $1952^{8}$ (ill. 1).

7. Hamidreza Ansari (dir.), Beaux-Arts 1940-1969, sélection de travaux d'étudiants architectes de la faculté des Beaux-Arts durant les trois premières décennies [trad. du titre persan], Téhéran, Honare memarie gharn, 2016. Si cette publication met à disposition des chercheurs une intéressante matière brute, la petite taille des reproductions et le manque d'information accompagnant les planches, notamment sur leur provenance, date et cadre de réalisation est à déplorer.

8. Sur le parcours de Seyhoun (Seihoun) aux Beaux-Arts voir le Dictionnaire des élèves architectes de l'école nationale supérieure des Beaux-Arts [en ligne] : https://agorha.inha.fr/inhaprod/ark:/54721/ 002156196 ; sur la construction du mausolée d'Avicenne : Talinn Grigor, Building Iran, New York, Periscope Publishing, 2009, p. 122-131. 


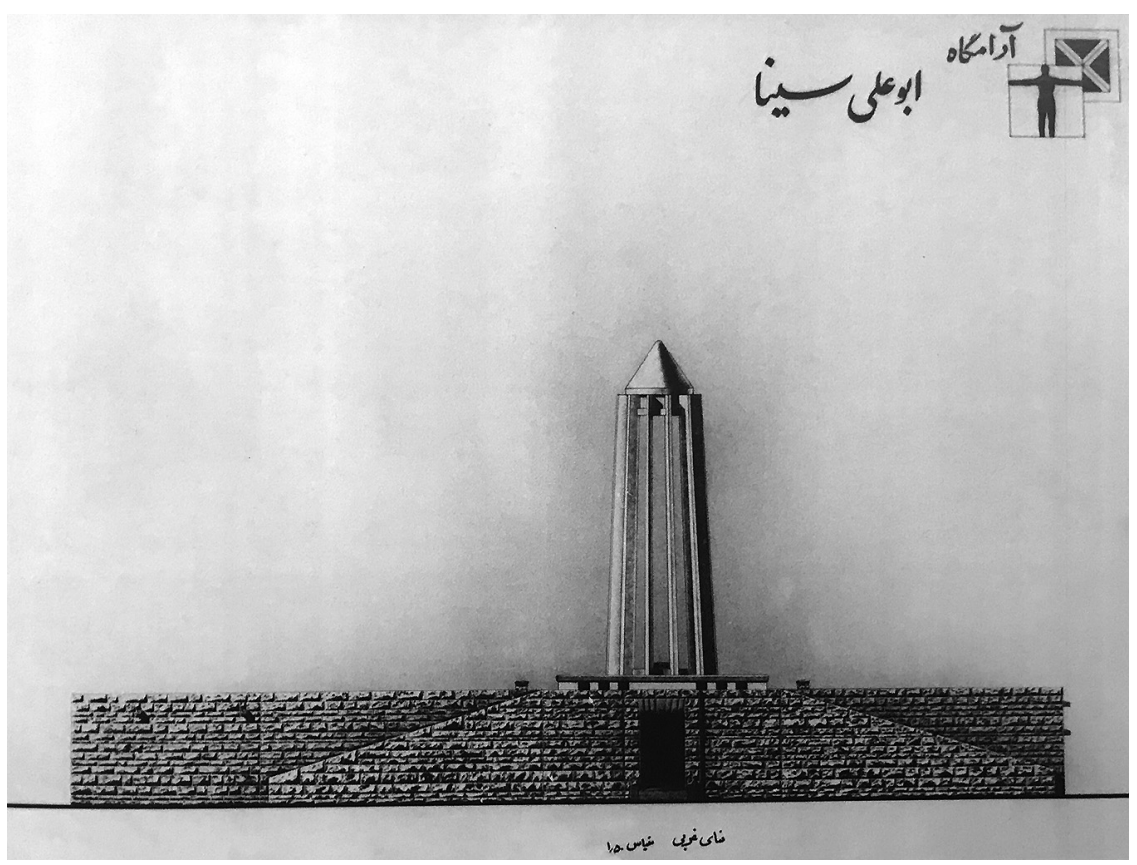

Ill. 1 : Mausolée d’Avicenne, Hamadan, Iran, Houshang Seyhoun arch., 1949-1952, élévation ouest, relevé réalisé par les étudiants en $1^{\text {re }}$ année de la Faculté des Beaux-Arts, n.d., (d'après Faculté des BeauxArts, sélection de travaux des étudiants architectes, 1940-1969, Téhéran, Honare memarie gharn, 2015, p. 68). (C) D.R.

\section{Présentation des planches de rendus}

Le français fait partie des cours obligatoires de la faculté dès l'ouverture jusqu'au début des années $1960^{\circ}$, sans doute en raison de la présence des professeurs français Maxime Siroux (1907-1975) et Roland Dubrulle. Mais l'enseignement se fait essentiellement en persan, surtout à partir du moment où les quatre ateliers sont confiés aux patrons iraniens. Pourtant, toutes les inscriptions et notices marquées sur les planches du concours de construction, dont le nombre pouvait atteindre une vingtaine, sont en français ou en anglais. Seul le titre de quelques rares projets apparaît en persan, et encore calligraphié, voire déformé dans un graphisme parfois à peine lisible. Sans doute l'alphabet latin est alors considéré plus compatible avec la forme des plans et le contenu des textes couchés sur les planches, puisque les élèves n'hésitent pas, en revanche, à recourir à l'écriture persane sur leurs rendus de relevé qui ont généralement pour objet l'architecture traditionnelle iranienne.

9. Asghar Saed Samiee, "Organisation du système pédagogique... ", op. cit., p. 33. 
Les années 1950 et 1960 correspondent à la substitution de l'anglais au français comme première langue étrangère en Iran, résultat de l'accroissement de l'influence américaine à la suite du coup d'état de $1953^{10}$. Cependant et malgré le rapatriement des professeurs français au lendemain de la Seconde Guerre mondiale, une bonne moitié des projets de construction de l'atelier Seyhoun est toujours renseignée en français. Cela ne saurait s'expliquer uniquement par la francophonie du professeur, puisque même dans l'atelier Mirfendereski, dont le patron est italophone, un projet sur trois est présenté en français. Cette langue reste donc appréciée et pratiquée par une part importante des étudiants architectes iraniens. Cela est corroboré par le fait que, malgré les critiques croissantes dans les années 1960, une quinzaine de diplômés et d'anciens élèves de la faculté téhéranaise vint parachever ses études à l'ENSBA ${ }^{11}$.

De même que la langue, les techniques de présentation du projet sont apprises en étudiant voire imitant " les planches des Beaux-Arts de Paris », en fait les recueils des concours d'architecture publiés par l'éditeur Vincent et Fréal ${ }^{12}$. Quelques appropriations de ces principes sont toutefois à remarquer. Le poché, le lavis, l'esthétique et le graphisme du dessin demeurent de rigueur, n'étant généralement récompensés de médaille que les projets parfaitement mis en forme selon les règles du rendu. L'ajout en revanche de quelques images de petite dimension, souvent photographies, parfois croquis, pour évoquer les références ou les sources d'inspiration du parti général, caractérise quelques-uns des rendus. Ce genre d'éléments totalement absent des planches de concours typiques de l'École parisienne, dévoile l'envers du décor et permet d'ouvrir le débat sur le processus de conception et les arguments du projet, autant sinon davantage que sur le résultat formalisé. Ceci révèle d'une part les attentes des élèves alors assoiffés de débats théoriques ${ }^{13}$, et annonce d'autre part, la voie dans laquelle la pédagogie de la faculté évolue par la suite, à savoir faire moins de projet mais en débattre davantage ${ }^{14}$.

10. Voir sur cet événement Homa Katouzian, Musaddiq and the Struggle For Power in Iran, Londres/ New York, I. B. Tauris, 1999.

11. Cf. Dictionnaire des élèves architectes de l'école nationale supérieure des Beaux-Arts, disponible en ligne : https://agorha.inha.fr/

12. Concours d'architecture de l'École nationale supérieure des Beaux-Arts, Paris, Vincent et Fréal, publications annuelles de 1906 à 1968. Ces recueils sont souvent cités comme modèle et outil pédagogique en usage dans les ateliers par les anciens élèves de la faculté de Téhéran : cf. Asghar Saed Samiee, "Organisation du système pédagogique... ", op. cit., p. 33 ; Manouchehr Tabibian, "La vie sociale en parallèle avec la carrière " [trad. du titre persan de l'entretien], Memari va farhang (Architecture et culture), revue trimestrielle, vol. 10, $\mathrm{n}^{\circ} 32,2008$, p. 39-40.

13. Iraj Etesam, "Technologie en parallèle avec l'enseignement traditionnel ", [trad. du titre persan de l'entretien], Memari va farhang (Architecture et culture), revue trimestrielle, vol. 10, $\mathrm{n}^{\circ} 32,2008$, p. 18.

14. Abdolhamid Eshragh, "La méthode d'enseignement en ateliers " [trad. du titre persan de l'entretien], Memari va farhang (Architecture et culture), revue trimestrielle, vol. 10, $\mathrm{n}^{\circ} 32,2008$, p. 24. 


\section{Programmes des projets de construction}

Le projet de construction est, aux débuts, encadré par l'architecte français Maxime Siroux qui applique la règle du concours, à savoir un programme commun défini par le professeur, pour tous les candidats ${ }^{15}$. À compter des années 1950 et de la prise du relai par le jeune diplômé de la faculté, Houshang Sanei, la liberté est accordée aux élèves de proposer chacun son propre programme à l'approbation du professeur $^{16}$. Ce dispositif estompe quelque peu le caractère concurrentiel de l'enseignement et élargit, en contrepartie, l'éventail des thèmes abordés par les élèves, même si les types d'édifices "classiques" restent les plus récurrents. En effet, sur une vingtaine de projets primés, on compte deux théâtres et un cirque, deux halles d'exposition et deux restaurants, un "monument " et des lieux sportifs : une tribune pour les compétitions nautiques, une piscine et un club de bowling. D'autres programmes, moins courants, révélant les intérêts personnels des étudiants, trouvent aussi place dans cet enseignement, comme le Cercle des Libanais à Téhéran, le Centre de recherche et d'observation des animaux ou encore une maison des soufis (Khanqah). On peut néanmoins remarquer que le lien avec l'actualité du pays est relativement faible, avec notamment l'absence, dans le lot, de bâtiments industriels alors que l'Iran entame une nouvelle phase d'industrialisation dans les années 1950 et surtout 1960 , grâce à l'augmentation du prix du pétrole ${ }^{17}$. De même, un seul projet consacré à l'habitat sur une vingtaine, tandis que la population iranienne double son taux de croissance pendant la même période ${ }^{18}$, ne manque pas d'interroger. Faudrait-il y voir le transfert, dans l'atelier d'un architecte DPLG, de l'une des traditions Beaux-Arts consistant à privilégier la grande architecture et les programmes de prestige ${ }^{19}$ ?

Pour les concours d'architecture, les patrons d'atelier rédigent à tour de rôle le programme et il leur arrive de faire traduire les sujets donnés à l'ENSBA de Paris pour les proposer, avec plus ou moins de décalage, à Téhéran ${ }^{20}$. Les étudiants iraniens ne sont donc pas, à cet égard, à l'abri de l'influence parisienne, surtout au sein d'un atelier dirigé par un ancien élève des Beaux-Arts. À titre de comparaison, les projets de l'atelier Mirfendereski - professeur formé à Florence - font ressortir des programmes un peu plus en phase avec l'air du temps : la faculté militaire à Ispahan, la résidence des experts en sciences spatiales dotée d'abri antiatomique sur

15. Hamidreza Ansari (dir.), Beaux-Arts 1940-1969, op. cit., p. 32 ; Iraj Etesam, «Technologie en parallèle avec l'enseignement traditionnel ", op. cit., p. 19.

16. Asghar Saed Samiee, "Organisation du système pédagogique... ", op. cit., p. 31.

17. Massoud Karshenas, Oil, State and Industrialization in Iran, Cambridge, Cambridge University Press, 1990.

18. Akbar Aghajanian, "Évolutions démographiques de l'Iran durant les cent dernières années " [trad. du titre persan], Kelk, no 57 , décembre 1995, p. 27-35.

19. Pour un exemple d'étude de programme des concours voir: Olivier Liardet, "Des phares au concours: de l'Académie royale d'architecture à l'École des Beaux-Arts 1745-1966 ", Livraisons de l'histoire de l'architecture, $\mathrm{n}^{\circ}$ 24, 2012, p. 32-61.

20. Asghar Saed Samiee, "Organisation du système pédagogique ... ", op. cit., p. 34. 


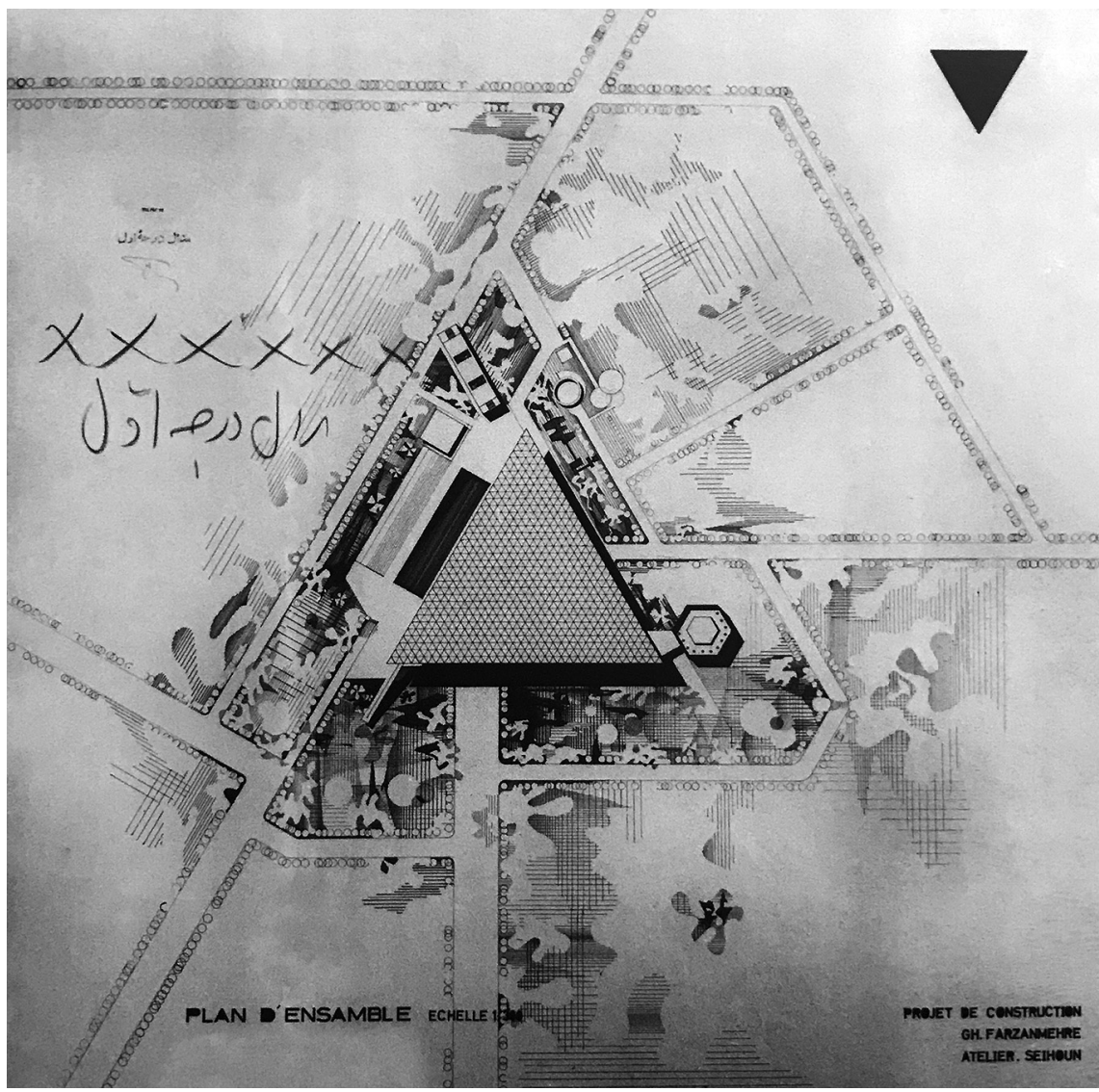

Ill. 2 : "Chemin de fer aérien Toutchale », Nord de Téhéran, projet de construction, élève M. Talaie, atelier Seyhoun, détails d'exécution des pylônes, n.d., seconde médaille avec félicitations du professeur (d'après Faculté des Beaux-Arts, op. cit., p. 145). (C) D.R.

le futur campus de Téhéran ou encore la préfabrication lourde dans le logement, à côté des sujets plus conventionnels comme un centre culturel, un aqueduc, une foire aux fleurs et des bains publics. Cela ne veut pas dire pour autant que tous les élèves de l'atelier Seyhoun soient insensibles aux besoins réels contemporains; les projets d'une station de téléphérique dans les montagnes du nord de Téhéran (ill. 2), celui d'une station de métro de la capitale de même qu'un ensemble d'habitats parasismiques à Ghazvin (ill. 3), ville à haut risque sismique, font écho aux programmes alors en étude et réflexion dans les administrations iraniennes. 


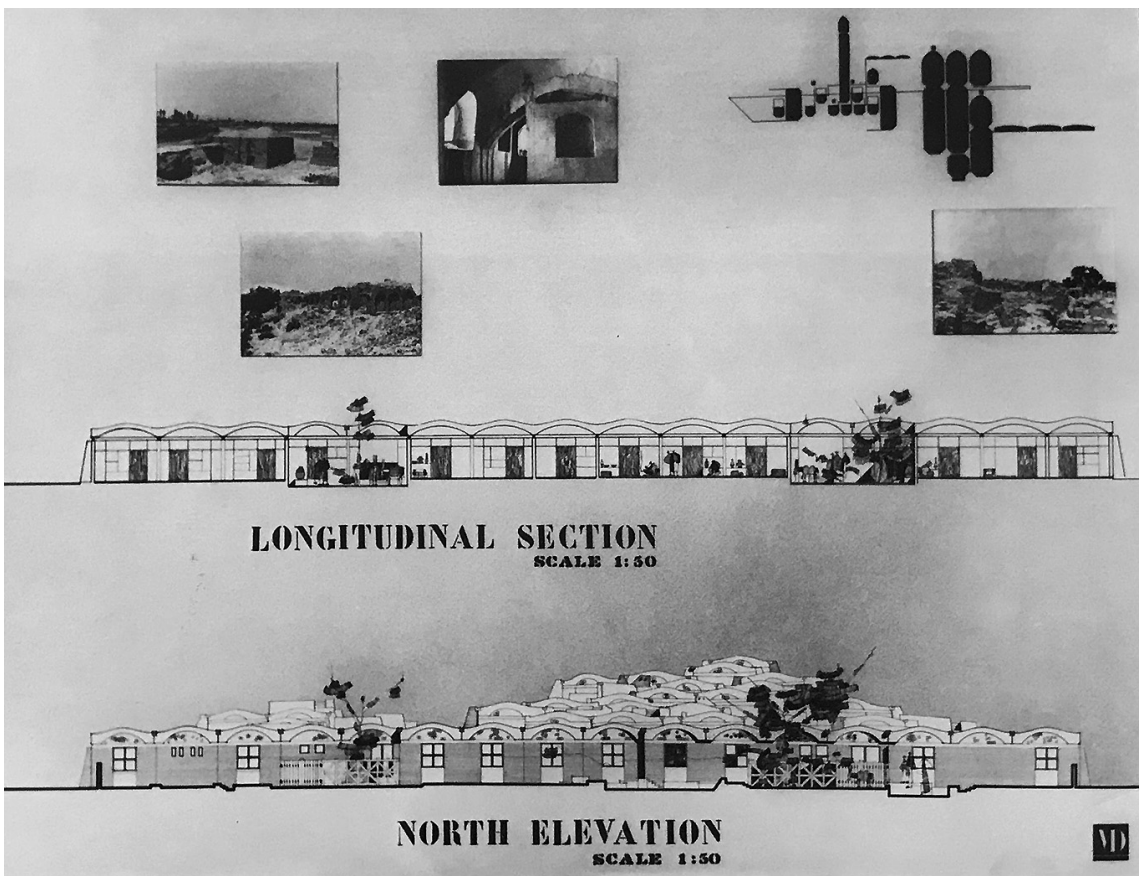

Ill. 3 : Ensemble d'habitats parasismiques à Ghazvin, Iran, élève Mahmoud Darvish, atelier Seyhoun, coupe longitudinale et élévation nord, n.d. ; à remarquer les photographies sur la planche présentant les références et le contexte géographique du projet (d'après Faculté des Beaux-Arts, op. cit., p. 111). (C) D.R.

\section{Insertion de l'édifice dans un site}

L'une des critiques récurrentes de l'enseignement de l'architecture aux BeauxArts concerne le peu d'attention accordé à l'insertion des projets dans un site réel ${ }^{21}$. Les plans sont généralement dessinés sur un canevas plus ou moins abstrait, souvent dans un vague contexte naturel, voire au milieu de nulle part. Ce constat vaut également pour les projets de construction de l'atelier Seyhoun. Les deux " meilleurs » rendus, ayant remporté l'un la première médaille, l'autre la même récompense augmentée des félicitations du jury, fait d'ailleurs très rare dans les concours de construction $^{22}$, sont à cet égard significatifs. Dans son projet pour une piscine et sauna, l'étudiant Gholamreza Farzanmehr propose un grand espace sur plan triangulaire couvert par une structure réticulaire, l'énorme prisme étant inséré, tel l'épicentre d'un aménagement paysager, dans un parcellaire vaguement dessiné (ill. 4).

21. Jacques Lucan, Composition, non-composition, architecture et théories au XIX et $X X^{e}$ siècles, Lausanne, Presses polytechniques et universitaires romandes, 2009, p. 123-124.

22. Cf. Abdolhamid Eshragh, "La méthode d'enseignement en atelier ", op. cit., p. 23 ; Asghar Saed Samiee, "Organisation du système pédagogique... ", op. cit., p. 32. 


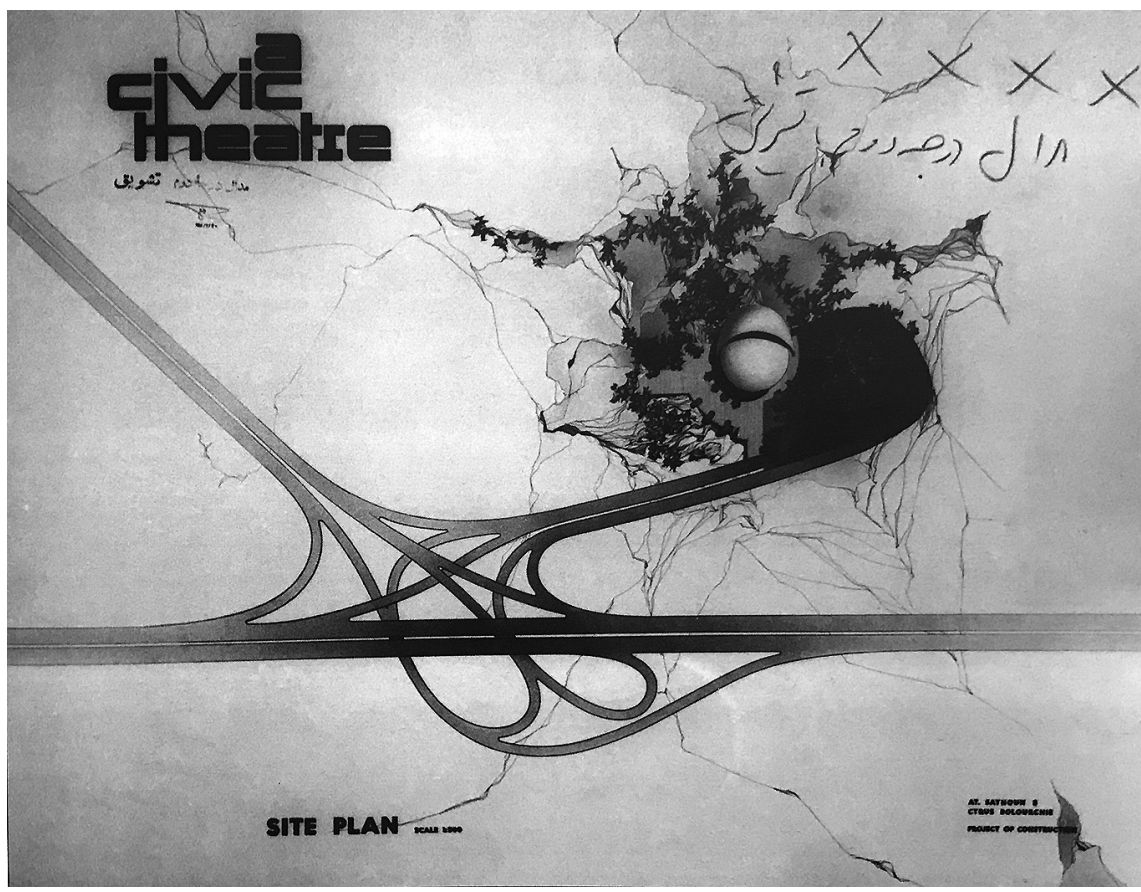

Ill. 4 : Piscine et Sauna, projet de construction, élève Gholamreza Farzanmehr, atelier Seyhoun, plan d'ensemble, n.d., première médaille (d'après Faculté des Beaux-Arts, op. cit., p. 242). (C) D.R.

Massoud Afsharmanesh, un autre élève de Seyhoun, ravit la première médaille et les félicitations du jury par un projet de maison de thé structurellement ambitieux : le plateau portant l'édifice est suspendu à un grand pylône métallique par une structure en câble et ferme en console, au-dessus du vide d'une vallée escarpée. Il est fort probable que ce projet a été conçu pour les côtes abruptes du nord de la capitale, terrain de randonnées et de balades des Téhéranais, et à ce titre investi par de nombreux cafés et maisons de thé. Cela dit, hormis une situation montagneuse imprécise, aucun élément sur les plans ne permet de connaître à coup sûr l'implantation géographique du projet, pis encore, le dessin sur une coupe transversale d'une voiture au pied du pylône de la structure rajoute de la confusion. Plus ironique est le projet d'un "théâtre de ville " dessiné par Cyrus Blourchi, récompensé d'une seconde médaille avec félicitations. La salle de spectacle ovoïde, doublée d'une scène machinée, est abritée dans une belle coque en béton armé, implantée non loin d'un échangeur, mais au milieu, dirait-on, d'une nature sauvage! (ill. 5). Mis à part ce cas exceptionnel de contraste évident entre l'intitulé du programme et la situation du projet évoquée par les dessins, on pourrait rétorquer que l'objectif de ce concours était avant tout de rendre compte de la compétence de l'étudiant à dessiner un projet jusque dans les détails constructifs en mobilisant les connais- 


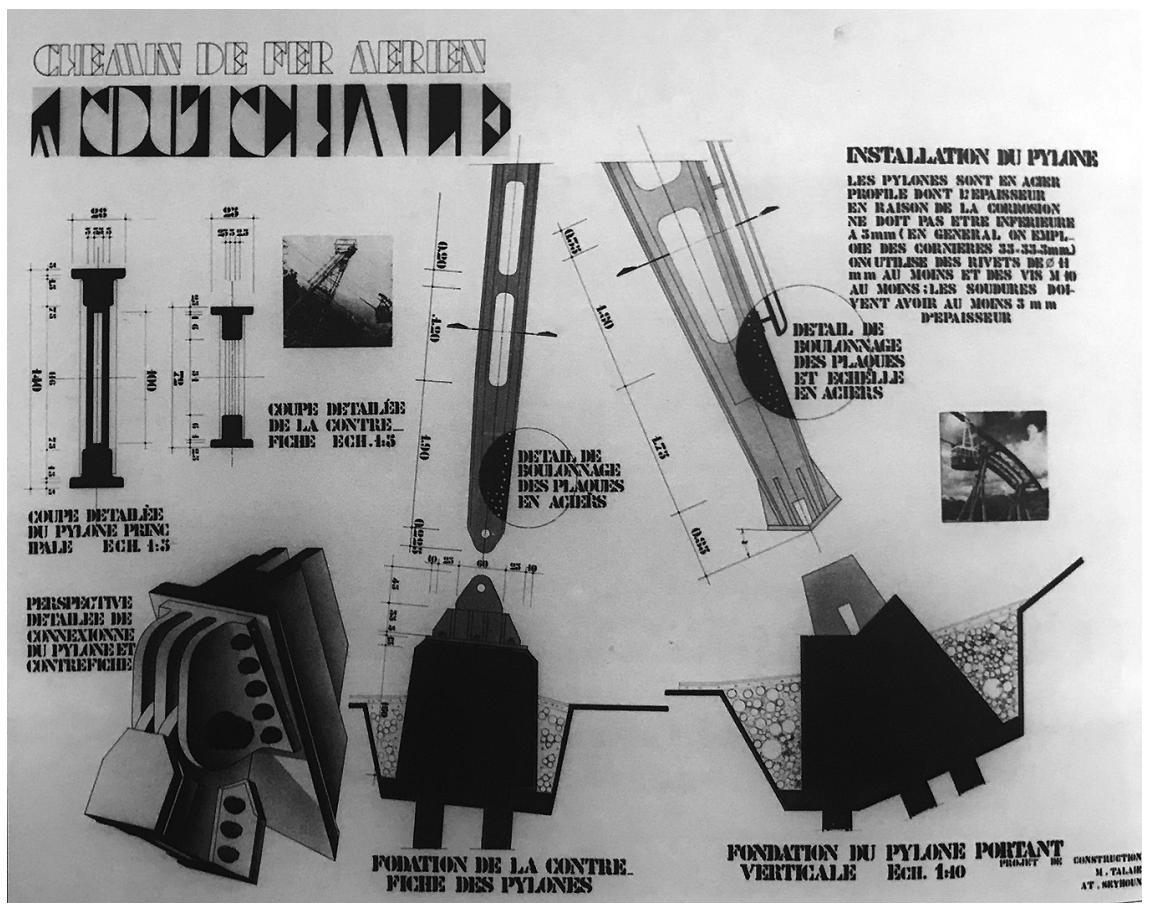

Ill. 5 : Un théatre de ville, projet de construction, élève Cyrus Bolourchi, atelier Seyhoun, plan de situation, n.d., seconde médaille avec félicitations (d'après Faculté des Beaux-Arts, op. cit., p. 166). (C) D.R.

sances acquises durant le premier cycle des études. Il n'en reste pas moins que l'insertion de l'édifice dans une situation réelle ne faisait donc pas partie des compétences à acquérir au cours de ce cursus.

\section{Conception technique et détails constructifs}

Malgré l'existence d'un cours de construction à l'ENSBA dès avant $1920^{23}$, dispensé et développé entre 1934 et 1962 par François Vitale ${ }^{24}$, les détracteurs du système Beaux-Arts ont souvent pointé la place marginale de l'enseignement des techniques, notamment constructives, dans la pédagogie de l'École parisienne, opposant cette dernière à celle plus vertueuse à cet égard des écoles polytechniques

23. Jean-Pierre Epron (dir.), Les Architectes et le projet, tome 2, Liège, Pierre Mardaga, 1992, p. 132.

24. Sébastien Di Vora, "Une histoire de l'enseignement de la construction en architecture : l'enseignement de François Vitale à l'École des Beaux-Arts ", mémoire de master, École d'architecture de Strasbourg, 2013 ; Amandine Diener, «La photographie au service de l'enseignement de la construction à l'École des Beaux-Arts. La démarche de François Vitale ", Livraisons d'histoire de l'architecture, $\mathrm{n}^{\mathrm{o}} 31,1^{\mathrm{er}}$ semestre 2016, p. 39-50. 

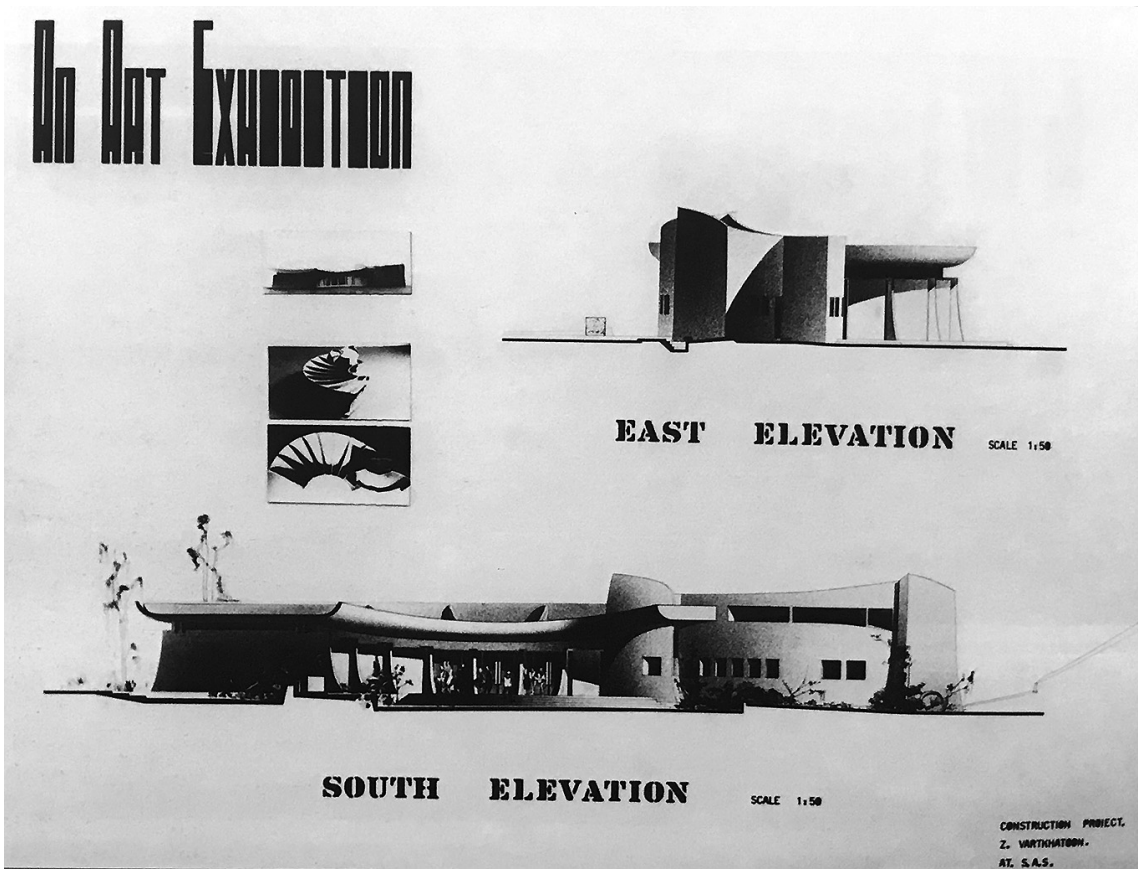

Ill. 6 : Une galerie d'art, projet de construction, élève Z. Vartkhatoon, atelier S.A.S., élévations est et sud, n.d., (d'après Faculté des Beaux-Arts, op. cit., p. 217). (C) D.R.

allemandes. À Téhéran, comme nous l'avons vu, la balance a basculé dès l'origine, et sous l'impulsion des architectes DPLG, du côté du modèle français. Or, la nomination, à la fin des années 1940 , d'un jeune professeur en charge des projets de construction était censée renforcer le volet technique de l'enseignement ${ }^{25}$. À travers les planches de l'atelier Seyhoun transparaît un effort plus ou moins grand pour penser les éléments et la mise en ouvre de l'édifice, des fondations à la toiture. Le trait commun à la majorité des projets reste, néanmoins, un parti structurel souvent monumental matérialisé par l'un des procédés constructifs peu ou prou innovants, à l'époque. Les structures suspendues en câbles, les fermes réticulaires, les voiles en béton armé, les toiles tendues et des coques plissées font partie des systèmes couramment adoptés (ill. 6). Il ne semble pas s'agir pour autant d'une course à la technologie au sein de l'atelier. Une certaine adéquation entre le programme et le parti structurel est généralement observée. Ainsi, lorsqu'il est question de concevoir un habitat rural résistant au tremblement de terre, une construction en briques armées est envisagée ; pour le Cercle des Libanais à Téhéran, un procédé de voûtes surbaissées, rappelant autant l'architecture traditionnelle iranienne que les maisons Jaoul de Le Corbusier achevées à la même époque. L'inspiration des réalisations du maître

25. Manouchehr Tabibian, "La vie sociale en parallèle avec la carrière ", op. cit., p. 40. 
moderniste ne se limite pas, d'ailleurs, au seul système constructif. On relève aussi l'imitation sinon la copie de ses ouvres les plus retentissantes, telle la chapelle de Ronchamp et la Cité radieuse de Marseille ; preuve que cette faculté, bien que fondée sur le modèle Beaux-Arts, n’était pas totalement hermétique aux idées et pratiques de l'un des plus fervents ennemis de l'École parisienne.

Alors que cette dernière explose sous les huées des contestataires de son présumé académisme, sa cadette téhéranaise connait également de grands changements sans que cela lui soit fatal : le personnel enseignant est régénéré par l'arrivée de jeunes diplômés iraniens d'autres universités européennes notamment italiennes; le système des ateliers est mis à bas, celui des concours progressivement abandonné au profit des cours théoriques étoffés ${ }^{26}$; Houchang Seyhoun prit sa retraite, ciblé par des protestations étudiantes comme figure emblématique et autoritaire d'une pédagogie démodée. Or, il semble plutôt l'adepte passionné d'un enseignement des BeauxArts iranisé, d'un système "éclectique, ni français ni italien " ${ }^{27}$ dont le "projet de construction " était l'une des caractéristiques: fruit du métissage du concours de construction et du projet de diplôme du système parisien, visant à armer les étudiants pour entrer dans la pratique professionnelle dès la fin d'un premier cycle d'études, dans un pays en pleine modernisation où la formation académique des architectes date à peine de trois décennies. Certains anciens élèves et enseignants de la faculté verront le revirement pédagogique de la fin des années 1960 comme salutaire pour l'enrichissement culturel et théorique des étudiants ${ }^{28}$; d'autres regretteront la perte définitive d'une pédagogie cohérente, solidaire et stable ${ }^{29}$, soulignant que nombre des meilleurs architectes iraniens des décennies suivantes étaient sortis de ces mêmes ateliers. L'histoire en jugera.

Shahram ABADIE Maître de conférences à l'École d'architecture de Clermont-Ferrand

26. Aliakbar Saremi, "Pensée moderne, architecture moderne ", [trad. du titre persan de l'entretien], Memari va farhang (Architecture et culture), revue trimestrielle, vol. 10, no 32, 2008, p. 53-55.

27. Ardeshir Cyrus, "Architecture au-delà de la construction " [trad. de titre persan de l'entretien], Memari va farhang (Architecture et culture), revue trimestrielle, vol. 10, nº 32, 2008, p. 27.

28. Iraj Etesam, "Technologie en parallèle avec l'enseignement traditionnel ", op. cit., p. 19; Darab Diba, "Action entre architecture et culture" [trad. de titre persan de l'entretien], Memari va farhang (Architecture et culture), revue trimestrielle, vol. 10, nº 32, 2008, p. 48.

29. Abdolhamid Eshragh, "La méthode d'enseignement en atelier ", op. cit., p. 25 ; Manouchehr Tabibian, "La vie sociale en parallèle avec la carrière ", op. cit., p. 40 ; Asghar Saed Samiee, "Organisation du système pédagogique... ", op. cit., p. 34. 